\title{
Crystallization Kinetics of Poly(3-Hydroxybutyrate) Granules in Different Environmental Conditions
}

\author{
Michael M. Porter, Jian Yu
}

Hawai'i Natural Energy Institute, University of Hawai'i at Mānoa, Honolulu, Hawai’i.

Email: jianyu@hawaii.edu

Received February $4^{\text {th }}, 2011$; revised May $4^{\text {th }} 2011$; accepted June $1^{\text {st }}, 2011$.

\begin{abstract}
Poly(3-hydroxybutyrate) (PHB) is a natural biopolyester accumulated in microbial cells as tiny amorphous granules. The nano- micro-particles have a variety of potential applications and behave differently in different environments. In this work, the in situ crystallization of native PHB granules was investigated under different environmental conditions. The isothermal crystallization kinetics of the granules was shown to follow Avrami's equation. The model parameter describing crystal growth is a function of temperature or $\mathrm{pH}$ and estimated from in situ crystallization measurements with attenuated total reflectance Fourier transform infrared (ATR-FTIR) spectroscopy. Empirical equations describing crystal growth are derived for the parameter values. $P H B$ granules heated at $80^{\circ} \mathrm{C}-140^{\circ} \mathrm{C}$ in acidic solution (pH 2) up to $4 \mathrm{hr}$ showed an increase in crystallinity from about 5\% to 35\% and moderate particle aggregation. PHB granules suspended in alkaline solutions ( $\mathrm{pH}$ 9-12) at room temperature up to $4 \mathrm{hr}$ showed an increase in crystallinity up to $45 \%$ and very little particle aggregation. It was found that the amorphousness of PHB granules in vivo is stabilized by water, lipids and proteins. Upon removal of these impurities, partial crystallization is induced which may inhibit extensive particle aggregation.
\end{abstract}

Keywords: Avrami Model, Crystallization Kinetics, PHA, PHB Granules, Biopolyester Particles

\section{Introduction}

Poly(3-hydroxybutyrate) (PHB) is a representative, naturally occurring bacterial polyhydroxyalkanoate (PHA) that can be produced from renewable feedstocks as an eco-friendly bioplastic [1,2]. In nature, PHB biopolyesters are accumulated in microbial cells as tiny intracellular amorphous granules $(0.2-0.5 \mu \mathrm{m}$ in diameter) (see Figure 1(a)) [1-5]. PHB granules can be extracted from microbial cells and purified by a number of different recovery processes [6-9]. During recovery, the environment of the amorphous granules changes and may cause the nano- micro-particles to crystallize [10-12]. Interestingly, in different environmental conditions the rate and extent of PHB crystallization varies, which may affect the degree of particle aggregation, resulting in different size and morphology of the biopolyester granules. Purified PHB particles may be useful in a variety of applications because PHB is completely biodegradable and biocompatible $[2,4,5]$.

The crystallization of PHB is an energetically favor- able process that may be induced by its environment Purified PHB granules become a semi-crystalline material ( $\sim 60 \%$ crystallinity) [13-15]. When pure PHB is heated above its melting point $\left(\sim 180^{\circ} \mathrm{C}\right)$, it becomes a fully amorphous melt $[14,16-20]$. Upon cooling, the PHB molecules crystallize, forming antiparallel helical chains linked by $\mathrm{C}-\mathrm{H} \cdots \mathrm{O}$ hydrogen bonds between the carbonyl $(\mathrm{C}=\mathrm{O})$ and methyl $\left(\mathrm{CH}_{3}\right)$ groups of the polyester backbone $[18,21,22]$. Crystallization begins as groups of amorphous molecules aggregate into tiny clusters forming nuclei $[19,23]$. Then, sheet-like structures known as lamellae grow radially outward from the nuclei in the form of spherulites $[19,23]$. The kinetics of PHB crystallization depend on the thermodynamics of phase change (i.e., amorphous to crystalline), the nature of nucleation and spherulitic crystal growth, and environmental factors such as temperature and chemical potential $[19,23,24]$.

In vivo, PHB granules are fully amorphous and surrounded by a monolayer membrane composed of phospholipids and proteins $[3,10,11,25]$. The amorphousness 
of native PHB in vivo is likely stabilized by a small amount of water $(5 \%-10 \%)$ contained within the granules and the monolayer membrane surrounding the granules $[2,3,10]$. In different environmental conditions, native PHB granules may undergo varying degrees of partial crystallization, which may be attributed to the removal of intra-granule water and the membrane lipids and proteins [10-12]. The rate and extent of in situ crystallization in the granules is a function of the environment, such as temperature or $\mathrm{pH}$. Understanding the crystallization kinetics of the PHB granules in situ is useful to help understand the processes of particle aggregation and the resulting size and morphology of the biopolyester particles.

This work shows that the in situ crystallization kinetics of native PHB granules in different environmental conditions can be modeled similar to that of pure PHB cooled from melt. The in situ crystallization of native PHB granules is shown to be induced or driven by the temperature or $\mathrm{pH}$ of the granule environment and dependent on environmental factors, such as granule impurities (i.e., non-PHB biomass and water) and particle morphology (i.e., aggregation). Such environmental factors may promote or inhibit PHB crystallization by changing the freeenergy barrier required for crystal growth or the number of nucleation sites available for crystal growth.

\section{Materials and Methods}

\subsection{PHB Granules}

In this work, Ralstonia eutropha cells containing $60-70$ $\mathrm{wt} \%$ PHB were stored at room temperature $\left(\sim 23^{\circ} \mathrm{C}\right)$ in solutions of $0.2 \mathrm{M}$ sulfuric acid $\left(\mathrm{H}_{2} \mathrm{SO}_{4}\right)$. The slurry contained $150-250 \mathrm{~g}$ dry cell mass/liter. Three aqueous solutions of PHB-containing cells were prepared as controlled samples of different crystallinity:

- Native amorphous PHB granules that were suspended in acidic solution (pH 2) as described above (see Figure 1(b));

- PHB granules heated in acidic solution with moderate crystallinity, where the native granule slurry was heated in glass test tubes at $80^{\circ} \mathrm{C}-140^{\circ} \mathrm{C}$ and maintained for 0 - $4 \mathrm{hr}$, then set at room temperature for several months for the apparent crystallinity to stabilize;

- PHB granules in solutions of varying $\mathrm{pH}$ with high crystallinity, where the $\mathrm{pH}$ of the native granule slurry was increased from 2 to 12 by adding equal volumes of varying concentrations of sodium hydroxide $(\mathrm{NaOH})$ to the native slurry at room temperature.

\subsection{Purified PHB}

Pure PHB was extracted from the microbial cells for comparison. The cells were freeze dried, suspended in

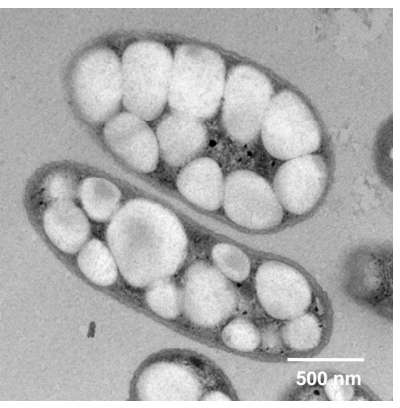

(a)

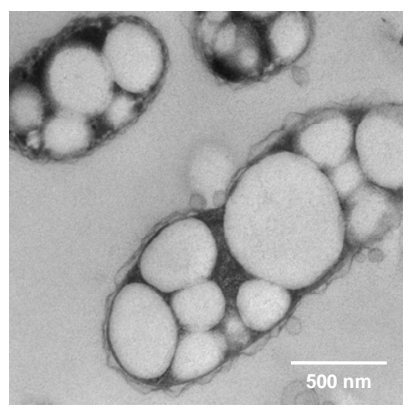

(b)
Figure 1. TEM images of PHB-containing cells: (a) in neutral solution (pH 7); (b) in acidic solution (pH 2). The bar is $500 \mathrm{~nm}$.

hot chloroform, and filtered to separate the polymer solution from the residual cell mass. Pure PHB was precipitated from the chloroform solution by adding hexanes. The precipitates were filtered, washed, and dried. The purified PHB was then dissolved in hot chloroform and cast on a clean glass surface as a thin film $(\sim 0.2 \mathrm{~mm}$ thickness).

\subsection{Attenuated Total Reflectance Fourier Transform Infrared Spectroscopy}

The infrared absorption spectra of the pure PHB and the PHB-containing cells were recorded with a Nicolet Avatar 370 FTIR spectrometer (Thermo Electron Co., Madison, WI). All measurements were taken in ambient conditions on a germanium crystal window of micro-horizontal attenuated total reflectance (ATR). A total of 32 scans were averaged for measurement of a single sample over $1 \mathrm{~min}$.

For time-resolved measurements describing the crystallization of pure PHB, the sample was first melted at $180^{\circ} \mathrm{C}$, then placed directly on the ATR window at 0 min and allowed to cool at room temperature $\left(\sim 23^{\circ} \mathrm{C}\right)$. Absorption spectra were collected every $1-2$ min over the duration $(30 \mathrm{~min}$ ) of the process. The crystallinity of PHB was monitored at the wavenumber $1184 \mathrm{~cm}^{-1}$ and scaled by the reference peak $1382 \mathrm{~cm}^{-1}$ according to crystalline measurements of pure PHA [21,26-28]. An absorption index $A I$ defined below (1) is a relative measurement of the degree of PHB crystallinity:

$$
A I=\frac{A_{1184}}{A_{1382}}
$$

where $A_{\tilde{v}}$ is the infrared absorption intensity of PHB at wavenumber $\tilde{v}$. The actual crystallinity of PHB was determined through a correlation of DSC measurements with the ATR-FTIR absorption spectra of three PHA samples of different crystallinity (data not shown here). The following linear correlation (2) was derived and used 
to calculate the crystallinity from the absorption index:

$$
X=-2.91 A I+3.04
$$

where $X$ is the apparent crystallinity of PHB.

For in situ measurements of the PHB-containing cells, a small drop $(\sim 2 \mu \mathrm{L})$ of aqueous solution was placed directly on the ATR window and allowed to evaporate at room temperature. During water evaporation, the PHBcontaining cells deposited onto the window via sedimentation and absorption spectra were collected every $1-2$ $\mathrm{min}$ for the duration $(30 \mathrm{~min}$ ) of the process. The absorption spectra were divided into three distinct stages: an initial scattering stage predominated by water absorption, a stable secondary stage representing the true, in situ crystallinity, and a final "artificial" crystallization stage caused by excess dehydration. The apparent, in situ crystallinity was measured during the stable, secondary stage of the measurement at the wavenumber $1184 \mathrm{~cm}^{-1}$, scaled by the reference peak $1382 \mathrm{~cm}^{-1}$ [21,26-28]. Spectral interference from background absorptions caused by water and other non-PHB cellular components were determined to be constant (data not shown here) and subtracted from the total absorption spectra. The in situ PHB crystallinity measurements were quantified using the same DSC/ATR-FTIR correlation mentioned above in (1) and (2).

\subsection{Transmission Electron Microscopy}

The transmission electron microscopy (TEM) images were viewed on a LEO 912 EFTEM (Zeiss, Germany) at $100 \mathrm{kV}$ and photographed with a frame-transfer CCD camera (Proscan, Germany). The cells were fixed with glutaraldehyde and calcium chloride in a sodium cacodylate buffer, then post-fixated with osmium tetroxide, stained with uranyl acetate, dehydrated with ethanol and embedded in epoxy. Ultrathin $(60-80 \mathrm{~nm})$ sections were obtained on an Ultracut E ultramicrotome (Reichert, Austria), double stained with uranyl acetate and lead citrate.

\subsection{Environmental Factors and Kinetics Modeling}

To determine the factors that may stabilize amorphous PHB granules, aqueous solutions of PHB-containing cells were dehydrated by freeze-drying and acetone extraction. The in situ crystallization behavior of the native granules in different environmental conditions was investigated by suspending PHB-containing cells in an acidic solution $(\mathrm{pH} 2)$ at high temperatures $\left(80^{\circ} \mathrm{C}-140^{\circ} \mathrm{C}\right)$ or in aqueous solutions of increasing $\mathrm{pH}(2-12)$ in ambient conditions.

The crystallization of pure PHB from melt was analyzed and compared to the in situ crystallization of the
PHB granules. Crystallinity measurements were obtained via ATR-FTIR and fit to Avrami's Equation (3) [21,28, 29]:

$$
X=1-\exp \left[-k t^{n}\right]
$$

where $X$ is the crystallinity, $t$ is the crystallization time, $k$ is a rate constant dependent on the formation of nuclei and spherulitic crystal growth, and $n$ is Avrami's exponent dependent on the nature of nucleation and geometry of crystal growth. Empirical equations to describe the growth rate parameter $(k)$ as a function of temperature or $\mathrm{pH}$ were derived. Based on the crystallization kinetics of polymers similar to $\mathrm{PHB}$, the growth rate parameter may be described by the Arrhenius Equation (4) as a function of temperature $[29,30]$ :

$$
k^{1 / n}=k_{o} \exp \left[-\frac{\Delta E_{a}}{R T}\right]
$$

where $\Delta E_{a}$ is the crystallization activation energy, $R$ is the gas constant $(8.314 \mathrm{~J} / \mathrm{K} \cdot \mathrm{mol}), k_{o}$ is a pre-exponential factor independent of temperature, and $T$ is the isothermal crystallization temperature in Kelvin. The growth rate parameters and Avrami's exponents were estimated by fitting the measured values to the empirical equations and discussed in terms of thermodynamic energy, granule geometry, spherulitic growth rate, and the nature of nucleation. Transmission electron microscopy (TEM) images of the granules in different environments were taken to show the extent of granule aggregation.

\section{Results}

\subsection{Crystallization of Pure PHB from Melt}

Figure 2(a) shows a time development of the infrared absorption spectra illustrating the crystallization of pure PHB cooled from an amorphous melt at $180^{\circ} \mathrm{C}$ (solid line) to a semi-crystalline solid at room temperature (dashed line) over $30 \mathrm{~min}$ in ambient conditions. As seen in the figure, the absorption intensity at $1184 \mathrm{~cm}^{-1}$ decreases with time, while the absorption intensity at $1382 \mathrm{~cm}^{-1}$ does not change with time and is taken as the reference peak [31]. Using the data from Figure 2(a) with (1) and (2), the corresponding crystallinity of PHB versus cooling time is plotted in Figure 2(b). Primary crystallization, which includes nucleation and spherulitic crystal growth, is represented by the solid data points and occurs between 2 - $15 \mathrm{~min}$. Secondary crystallization, or the rearrangement of PHB molecules into more energetically favorable structures, is represented by the hollow data points and occurs after $15 \mathrm{~min}$. The initial data points $(0$ $2 \mathrm{~min})$ are neglected in this analysis because the thermal history of the sample may be unstable and is not known. 


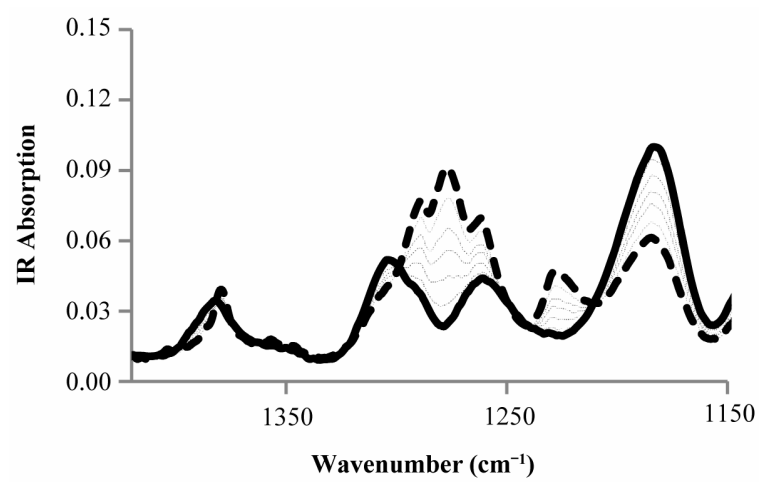

(a)

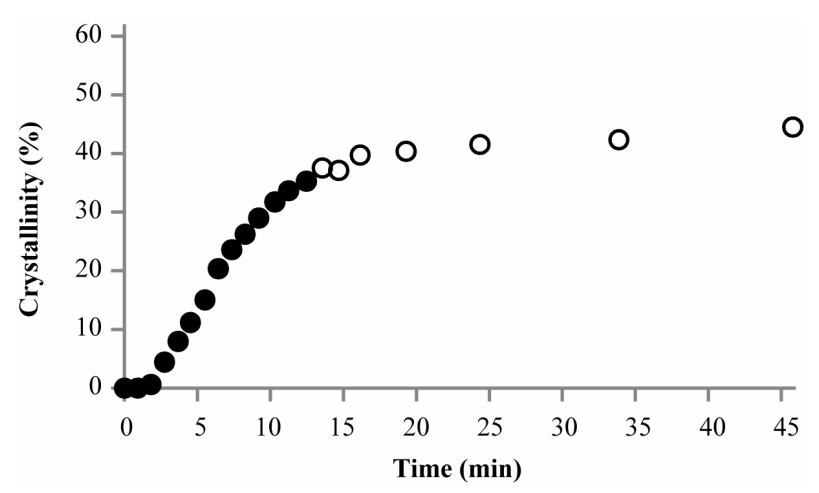

(b)

Figure 2. (a) Time development of the infrared absorption spectra of pure PHB, illustrating the crystallization due to cooling from an amorphous melt at $180^{\circ} \mathrm{C}$ (solid line) to a semi-crystalline solid at room temperature (dashed line); (b) Crystallinity versus cooling time of pure PHB cooled from melt at $180^{\circ} \mathrm{C}$ to room temperature, illustrating primary crystallization (solid data points) and secondary crystallization (hollow data points).

\subsection{Crystallization of Native PHB Granules}

The native PHB-containing cells stored in acidic solution (pH 2) exhibited no biological activity. The mild acidic conditions caused some damage to the cell walls as seen in Figure 1(b), but did not significantly change the nature of the native PHB granules.

Figure 3(a) shows a time development of the infrared absorption spectra of the native PHB granules in microbial cells suspended in acidic solution. In 30 min of measurement, a spectral pattern is observed that resembles the crystallization of pure PHB seen in Figure 2(a). Initially, the absorption spectrum of the solution is dominated by water (dotted line). As water evaporates in approximately $5 \mathrm{~min}$, a secondary spectrum of the PHB granules and other cellular components emerges due to sedimentation (solid line). This spectrum represents the true, instantaneous crystallinity of the native granules. After $15 \mathrm{~min}$, the spectra changes due to excess dehydra- tion, becoming fully developed, but no longer represents the true, instantaneous crystallinity of the native granules (dashed line).

The in situ crystallinity of the native PHB granules versus measurement time corresponding to Figure 3(a) is plotted in Figure 3(b). As mentioned, the instantaneous crystallinity was measured during the stable secondary stage (5 - $15 \mathrm{~min}$ ), after the initial scattering stage predominated by water $(0-5 \mathrm{~min})$, and before the final artificial stage due to excess dehydration (15 - $30 \mathrm{~min})$. After 15 - 30 min the PHB crystallinity increased up to $32 \%$, corresponding to a water content of less than $20 \%$. The true, instantaneous crystallinity of the native granules averaged over the secondary stage (5 - $15 \mathrm{~min}$ ) is $4.89 \% \pm$ $1.84 \%$.

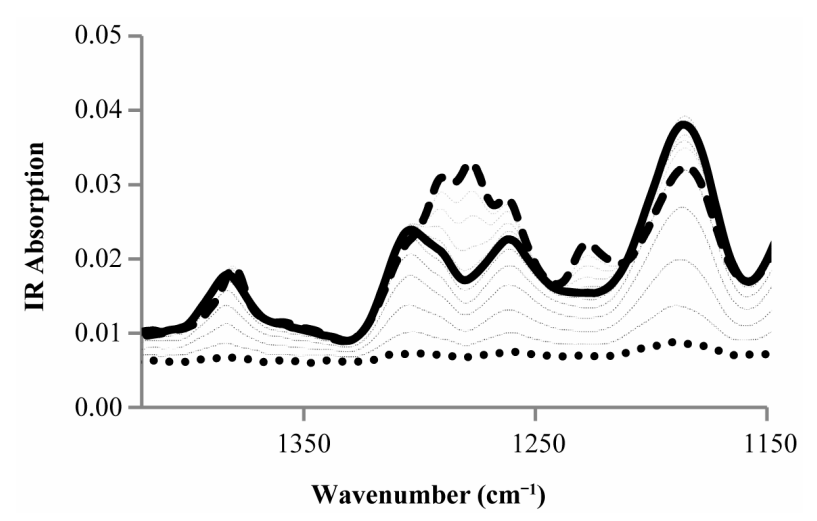

(a)

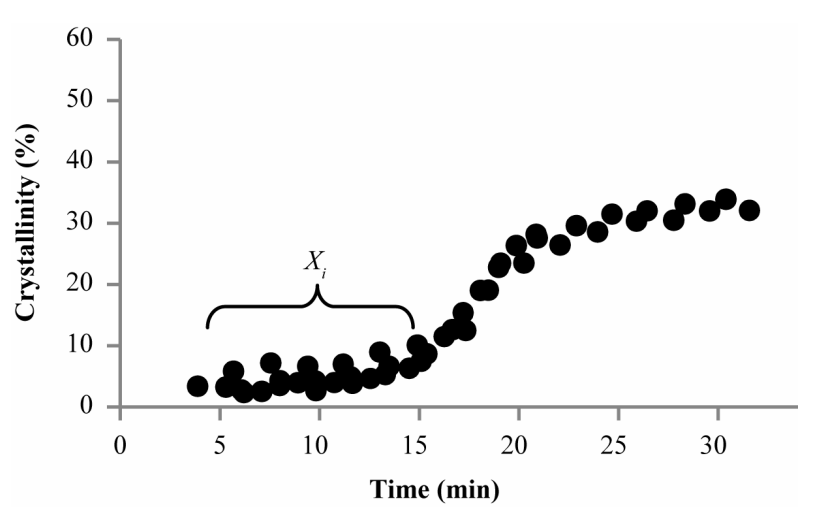

(b)

Figure 3. (a) Time development of the infrared absorption spectra of PHB-containing cells suspended in acidic solution (pH 2), illustrating the initial spectrum dominated by water at $\mathbf{0}$ mins (dotted line), the secondary spectrum characteristic of the instantaneous crystallinity after 10 mins (solid line), and the final spectrum caused by extensive dehydration after 30 mins (dashed line); (b) In situ crystallinity versus measurement time for native PHB granules in acidic solution at room temperature, illustrating the instantaneous crystallinity at $5-15$ mins $\left(X_{i}=4.89 \pm 1.84 \%\right)$. The final crystallinity $(32 \%)$ is attributed to excess dehydration. 
In further analysis, dehydration of the PHB-containing cells was observed to induce partial crystallization in the native granules. Cells freeze-dried for $3 \mathrm{hr}$ exhibited a slow crystallization of PHB, increasing up to $33 \%$ over the first $24 \mathrm{hr}$ after drying. In contrast, cells dehydrated with acetone extraction showed a much faster crystallization of PHB up to $52 \%$ after $24 \mathrm{hr}$. The increased crystallinity observed from acetone extraction is attributed to the simultaneous removal of water, as well as, lipids from the granules.

\subsection{Crystallization of PHB Granules Subjected to High Temperatures}

PHB-containing cells heated to and maintained at $80^{\circ} \mathrm{C}$ $140^{\circ} \mathrm{C}$ for $0-4 \mathrm{hr}$ in acidic solution ( $\left.\mathrm{pH} 2\right)$ showed an increase in PHB crystallization and granule aggregation with increasing temperature and exposure time. A significant increase in crystallinity observed $(20 \%-35 \%)$ in the granules heated above $80^{\circ} \mathrm{C}$ suggests that heating the cell slurry may have induced partial crystallization where intra-granule water, proteins and lipids were displaced from the PHB particles. Figure 4(a) contains a representative TEM image of the granules heated at $120^{\circ} \mathrm{C}$ for 1 $\mathrm{hr}$ in acidic solution $(\mathrm{pH} 2)$. As seen in the figure, heating the PHB-containing cells induced moderate granule aggregation. The instantaneous crystallinity of the PHB granules at $120^{\circ} \mathrm{C}$ for $1 \mathrm{hr}$, for instance, reached $22.52 \%$.

\subsection{Crystallization of PHB Granules Subjected to Varying pH}

Raising the $\mathrm{pH}$ of the PHB-containing cells from 2 to 12 in ambient conditions showed an increase in granule crystallinity with increasing $\mathrm{pH}$. Increasing the $\mathrm{pH}$, however, did not seem to induce granule aggregation and the in situ crystallization of the PHB granules seemed to occur instantaneously. Most likely, increasing the $\mathrm{pH}$ may have dissolved lipids and proteins from the granule membranes, inducing a quick partial crystallization at the particle surfaces where the granule membranes were removed. Figure 4(b) contains a representative TEM image of the granules at $\mathrm{pH} 12$ for $1 \mathrm{hr}$ in room temperature. As seen in the figure, increasing the $\mathrm{pH}$ of the PHB-containing cells induced very little granule aggregation. The instantaneous crystallinity of the PHB granules at $\mathrm{pH} 12$ for $1 \mathrm{hr}$, for instance, reached $40.59 \%$.

\subsection{Modeling the Crystallization Kinetics of PHB}

The crystallization of PHB is well described by Avrami's Equation (3) for isothermal crystallization, and is conveniently rewritten in linear form (5) $[21,28,29]$ :

$$
\log (-\ln (1-X))=\log (k)+n \log (t)
$$

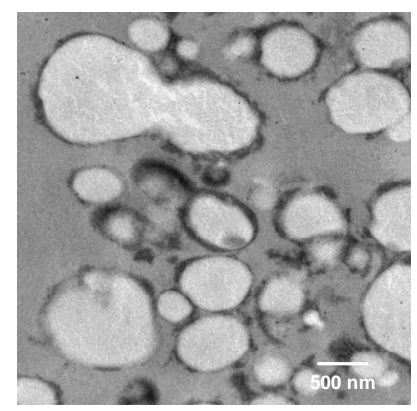

(a)

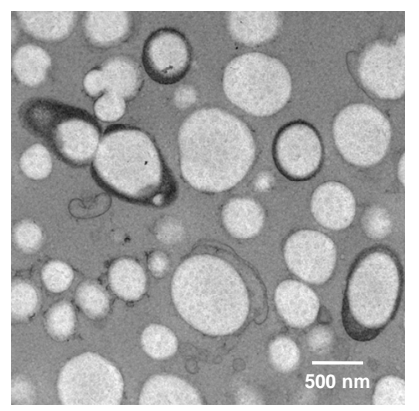

(b)
Figure 4. TEM images of PHB granules. (a) heated in acidic solution (pH 2) at $120^{\circ} \mathrm{C}$ for $1 \mathrm{hr}$; (b) suspended in alkaline solution (pH 12) at room temperature for $1 \mathrm{hr}$. The bar is $500 \mathrm{~nm}$.

Referring to Figure 2(b), the primary crystallization stage (2 - $15 \mathrm{~min})$ of pure PHB can be fit to Avrami's equation by plotting $\log (-\ln (1-X))$ versus $\log (t)$. Figure 5 contains a log-log plot for the crystallization of pure PHB from melt. Numerical values of Avrami's exponent and the growth rate parameter were obtained from the slope and intercept to be $n=1.5010$ and $k=0.0118$, respectively. When compared to published results, these values are close to the documented range $(n=1.58-1.92$; $k=0.04-0.13$ ) for the isothermal crystallization of neat PHB [31].

Similar to the crystallization of pure PHB, the in situ crystallization of PHB granules subjected to heating or varying $\mathrm{pH}$ can be modeled by Avrami's Equation (3), where $X$ is the in situ crystallinity of the PHB granules and $t$ is the period of time the granules are exposed to the different environmental conditions.

Table 1 gives the values of $n$ and $k$ obtained from linear correlations of the crystallinity data from the Avrami analysis of the PHB granules heated at $80^{\circ} \mathrm{C}-140^{\circ} \mathrm{C}$ for $0-4 \mathrm{hr}$ in acidic solution (pH 2). Table 1 also shows the measured in situ crystallinity of the granules after $4 \mathrm{hr}$ of heating at each temperature. From observation it is apparent that Avrami's exponent remains constant with temperature, so that $n=0.2140$. Further analysis shows that the crystallization growth rate parameter increases with temperature and may follow the Arrhenius equation previously mentioned (4). Rewriting the Arrhenius equation in linear form $(6)[29,30]$ :

$$
\frac{1}{n} \ln (k)=\ln \left(k_{o}\right)-\frac{\Delta E_{a}}{R}\left(\frac{1}{T}\right)
$$

and plotting $(1 / n) \ln (k)$ versus $1 / T$, as seen in Figure 6, the pre-exponential factor and activation energy were found from the linear correlation to be $k_{o}=78.73 \mathrm{~min}^{-1}$ and $\Delta E_{a}=48.82 \mathrm{~kJ} / \mathrm{mol}$, respectively. When compared to the activation energy of polypropylene $(163 \mathrm{~kJ} / \mathrm{mol})$, 
Table 1. Crystallization of PHB granules heated in acidic solution (pH 2).

\begin{tabular}{ccccc}
\hline$T\left({ }^{\circ} \mathrm{C}\right)$ & $X(\%)$ after $4 \mathrm{hr}$ & $n$ & $k\left(\mathrm{~min}^{-1}\right)$ & $R^{2}$ \\
\hline 25 & 4.89 & --- & --- & --- \\
80 & 21.00 & 0.2159 & 0.0711 & 0.9915 \\
100 & 23.26 & 0.1922 & 0.0904 & 0.9806 \\
120 & 27.19 & 0.2018 & 0.1042 & 0.9274 \\
140 & 35.54 & 0.2459 & 0.1200 & 0.9754 \\
AVG & --- & 0.2140 & --- & --- \\
STD & --- & 0.0234 & --- & --- \\
\hline
\end{tabular}

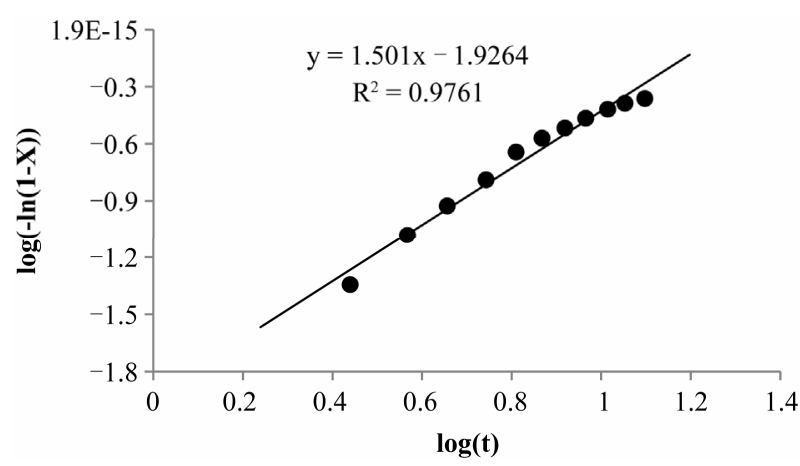

Figure 5. Determination of the crystallization rate constant $\boldsymbol{k}$ and the Avrami exponent $\boldsymbol{n}$ for the crystallization of pure PHB at room temperature during primary crystallization (2 - 15 min).

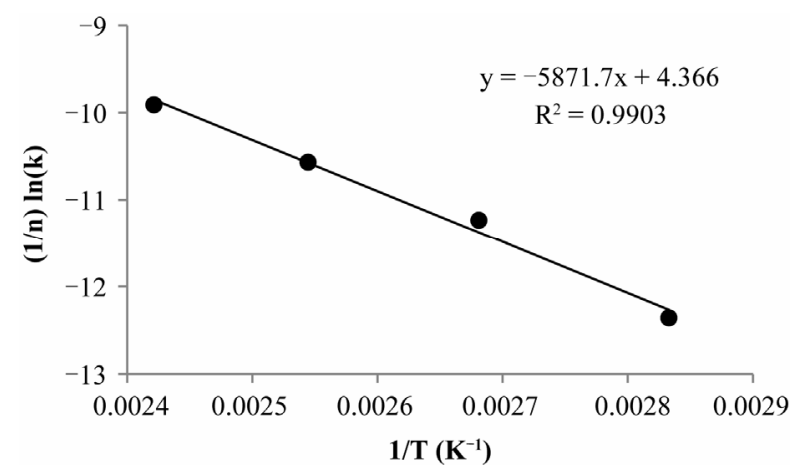

Figure 6. Crystallization growth rate parameter $k$ as a function of temperature for heated PHB granules.

the activation energy determined for the heated PHB granules $(48.82 \mathrm{~kJ} / \mathrm{mol})$ is much lower, but of similar magnitude [29].

Table 2 contains the values of $n$ and $k$ obtained from linear correlations of the crystallinity data from the Avrami analysis of the PHB granules suspended in solutions of varying $\mathrm{pH}(2-12)$ for $0-4 \mathrm{hr}$ at room temperature. Table 2 also shows the in situ crystallinity of the PHB granules averaged over the $4 \mathrm{hr}$ of exposure time.
The value of the Avrami exponent ( $n$ ) was found to be around zero. As previously mentioned, raising the $\mathrm{pH}$ of the cell slurry caused the PHB granules to crystallize nearly instantaneously. Therefore, the in situ crystallinity was assumed to be independent of the exposure time. Figure 7 contains a plot of the term $\log (-\ln (1-X))$ from Avrami's equation versus pOH $(=14-\mathrm{pH})$. From the log plot in Figure 7, it is apparent that the PHB crystallinity is linearly correlated to the $\mathrm{pOH}$ of the cell slurry, such that:

$$
\log (-\ln (1-X))=\alpha(\mathrm{pOH})+\beta
$$

where the constants $\alpha=-0.0891$ and $\beta=-0.1219$. Then combining (5) and (7) with the observation that $N=$ 0 , the growth rate parameter is expressed as:

$$
k=\left(10^{\beta}\right)\left[\mathrm{OH}^{-}\right]^{\alpha}
$$

where $\left[\mathrm{OH}^{-}\right]$is the concentration of hydroxide ions in the solution. No temperature dependence appears in this empirical relation because the $\mathrm{pH}$ of the granules was adjusted at a constant room temperature $\left(\sim 23^{\circ} \mathrm{C}\right)$.

Table 3 outlines the kinetics models describing the crystallization of pure PHB cooled from melt, PHB granules heated in acidic solution, and PHB granules of

Table 2. Crystallization of PHB granules in solutions of varying $\mathrm{pH}$ at room temperature $\left(\sim 23^{\circ} \mathrm{C}\right)$.

\begin{tabular}{cccc}
\hline $\mathrm{pH}$ & $X \pm \mathrm{STD}(\%)$ & $n$ & $k\left(\mathrm{~min}^{-1}\right)$ \\
\hline 2.00 & $6.99 \pm 1.41$ & 0.00 & 0.0645 \\
3.55 & $7.61 \pm 1.36$ & & 0.0886 \\
9.30 & $26.70 \pm 0.52$ & & 0.2881 \\
11.06 & $28.43 \pm 2.65$ & & 0.4133 \\
11.80 & $41.14 \pm 1.96$ & & 0.4811 \\
12.12 & $41.25 \pm 4.63$ & $\Downarrow$ & 0.5137 \\
\hline
\end{tabular}

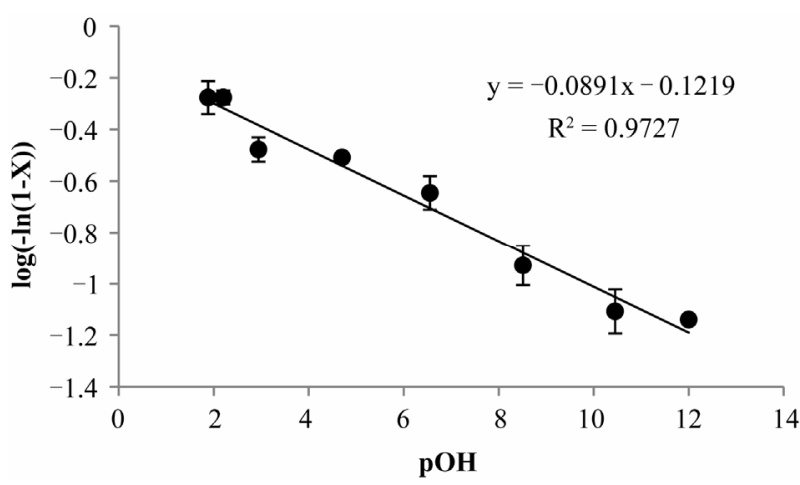

Figure 7. Log plot illustrating the linearity of in situ crystallization versus pOH of PHB granules suspended in solutions of varying $\mathrm{pH}$. 
Table 3. Crystallization models of pure PHB and PHB granules in different environmental conditions by Avrami analysis.

\begin{tabular}{|c|c|c|c|}
\hline & Pure PHB & PHB Granules (Heat) & PHB Granules $(p H)$ \\
\hline Crystallinity & $X=1-\exp \left[-k t^{n}\right]$ & $X=1-\exp \left[-k t^{n}\right]$ & $X=1-\exp \left[-k t^{n}\right]$ \\
\hline \multirow[t]{2}{*}{ Growth Rate Parameter } & $k^{1 / n}=k_{o} \exp \left[-\frac{\Delta E_{a}}{D T}\right]$ & $k^{1 / n}=k_{o} \exp \left[-\frac{\Delta E_{a}}{R T}\right]$ & $k=\left(10^{\beta}\right)\left[\mathrm{OH}^{-}\right]^{\alpha}$ \\
\hline & & $k_{o}=78.73 \mathrm{~min}^{-1} ; \Delta E_{a}=48.82 \mathrm{~kJ} / \mathrm{mol}$ & $\alpha=-0.0891 ; \quad \beta=-0.1219$ \\
\hline$n$ & 1.5010 & 0.2140 & 0 \\
\hline$k\left(\min ^{-1}\right)$ & 0.0118 & $0.0725-0.1215^{\mathrm{a}}$ & $0.0428-0.7553^{\mathrm{b}}$ \\
\hline $\mathrm{R}^{2}$ & 0.9761 & 0.9903 & 0.9727 \\
\hline
\end{tabular}

${ }^{\mathrm{a}}$ Range of $k$ for temperatures $80^{\circ} \mathrm{C}-140^{\circ} \mathrm{C}$; ${ }^{\mathrm{b}}$ Range of $k$ for $\mathrm{pH} 0-14$.

varying $\mathrm{pH}$. Table 3 also includes numerical values of the empirical constants, Avrami exponents $(n)$, and growth rate parameters $(k)$ determined for each of the three cases.

\section{Discussion}

\subsection{Stabilizing Factors of Amorphous PHB Granules}

According to the observation on dehydration of the native PHB granules, removal of water from the granules, as well as, removal of lipids from the granule membranes induced some partial crystallization. It is widely accepted that PHB granules are fully amorphous in vivo having a crystallinity of $0 \%$ in a neutral culture medium $(\mathrm{pH} 7)$ [3, 10]. When partially isolated and/or subjected to varying environmental conditions, however, the PHB granules undergo varying degrees of in situ crystallization [10-12]. As expected, the mild acidic solution ( $\mathrm{pH}$ 2) used to store the PHB-containing cells triggered very minuscule changes in crystallinity $(\sim 5 \%)$. It seems that the intragranule water and granule membranes remained largely intact, stabilizing the native PHB granules in a mostly amorphous state. Furthermore, the crystallization that occurred due to excess dehydration during ATR- FTIR measurement and dehydration by freeze-drying the cells did not exceed $35 \%$. Hence, the remaining granule membranes obviously kept the PHB granules from reaching a fully crystalline state $(\sim 60 \%)$. When dehydrated with acetone, on the other hand, the native granules became highly crystalline $(\sim 52 \%)$ in $24 \mathrm{hr}$, further suggesting that the lipids dissolved in acetone stabilized the native granules in a prior amorphous state.

The crystallization of $\mathrm{PHB}$ is primarily driven by $\mathrm{C}-$ $\mathrm{H} \cdots \mathrm{O}$ hydrogen bonding between the $\mathrm{C}=\mathrm{O}$ and $\mathrm{CH}_{3}$ groups in PHB [18,21,22]. Weakening the hydrogen bonds by melting or disrupting the hydrogen bonds with environmental impurities may inhibit the crystallization of PHB [10,13-15]. As noted, the amorphousness of native PHB granules in vivo must be stabilized by the monolayer membrane surrounding the particles and intra-granule water $[2,3,10]$. The $5 \%-10 \%$ of water present in the granules is thought to act as a plasticizer, forming hydrogen bonds with the $\mathrm{C}=\mathrm{O}$ and $\mathrm{CH}_{3}$ groups of the biopolyester backbones [10]. These hydrogen bonds prevent strong intra- and intermolecular interactions from occurring between the $\mathrm{C}=\mathrm{O}$ and $\mathrm{CH}_{3}$ groups in PHB $[3,10,12]$. Similarly, the granule membranes prevent strong interactions from occurring between PHB molecules in adjacent granules. Removal of the intra-granule water or the granule membrane promotes in situ crystallization due to increased $\mathrm{C}-\mathrm{H} \cdots \mathrm{O}$ hydrogen bonding, which is analogous to the cooling crystallization process in pure PHB [10]. If there is very little or no granule crystallization, regions of particles without granule membranes tend to aggregate.

\subsection{Interpretation of Avrami Exponents and Growth Rate Parameters}

Table 3 contains the equations and empirical constants determined from the Avrami analyses for the crystallization of pure PHB, PHB granules heated in acidic solution $(\mathrm{pH} 2)$, and $\mathrm{PHB}$ granules in solutions of varying $\mathrm{pH}$ at room temperature. As previously shown, Avrami's equation can be used to describe the crystallization of pure PHB, as well as, the in situ crystallization of native PHB granules in different environmental conditions. Differences in the Avrami exponents $(n)$ and the growth rate parameters $(k)$ for each condition provide the insight necessary to understand the different crystallization processes. To help visualize the geometric nature of crystallization, it is useful to introduce a theoretical size parameter $(w)$ describing crystal growth:

$$
w=\frac{r_{n}}{r_{s}}
$$


and the critical nuclei radii $\left(r_{c r}\right)$ required to overcome the free energy barrier for nucleation $[23,24]$ :

$$
r_{c r}=-\frac{2 \sigma}{\Delta G_{v}}
$$

where the average radii of the nuclei and corresponding spherulites are $r_{n}$ and $r_{s}$, and the surface and volumetric energies associated with nucleation are $\sigma$ and $\Delta G_{v}$, respectively.

The Avrami exponent $(n)$ is a measure of the geometric constraints associated with isothermal crystallization, describing the type of crystal growth (i.e., geometry of spherulites) and the nature of nucleation (i.e., number and size of nucleation points). A large Avrami exponent $(n>1)$ signifies relatively large spherulite growth from infinitesimal, point-source nuclei ( $w \rightarrow 0)$, as seen in the observation of pure PHB where $n=1.5010$. Because pure PHB is a neat homopolymer, it undergoes favorable, homogeneous nucleation, in which volumetric energy dominates, so that $r_{n}>r_{c r}$ [23,24]. A small Avrami exponent $(n<1)$, represents small spherulite growth from tiny, surface-source nuclei $(0 \leq w<1)$, as seen in the heated granules where $n=0.2140$ and the granules at high $\mathrm{pH}$ where $n=0$. This heterogeneous nucleation phenomenon most likely occurs at interfacial surfaces created by the removal of non-PHB biomass and water, lowering the free energy barrier and allowing a greater number of smaller nuclei to crystallize, so that $r_{n}>r_{c r}$. As the Avrami exponent approaches zero $(n \rightarrow 0)$, the size of spherulites may be restricted by material availability (i.e., amount of PHB per granule) or the impingement of adjacent crystallites, and unable grow beyond the size of the initial nuclei $(w \rightarrow 1)$. This behavior is seen in the granules at high $\mathrm{pH}$ where $n=0$, which is indicative of nearly instantaneous, time independent crystal growth where crystalline regions are composed of several tiny crystallites roughly the size of individual nuclei.

The growth rate parameter $(k)$ is a measure associated with the frequency of nucleation (i.e., number of nuclei) and subsequent spherulitic crystal growth. That is, a material exhibiting a small growth rate parameter may crystallize from very few nucleation points into relatively large spherulites. Conversely, a material exhibiting a larger growth rate parameter may crystallize from several nucleation points into many tiny spherulites. As seen in Tables 1-3, the growth rate parameter is smallest for pure PHB where $k=0.0118$ and increases with increasing temperature $(k \rightarrow 0.1215)$ and increasing $\mathrm{pH}$ $(k \rightarrow 0.7553)$. This suggests that, although the average growth rate of an individual spherulite of PHB may not differ in varying environments, the overall crystallization growth rate of PHB is affected by the frequency of nucleation; which, in pure PHB is significantly less than that of the heated granules and the granules at high $\mathrm{pH}$. The growth rate parameter describing the heated granules increases with temperature most likely because heterogeneous nucleation is more favorable at higher temperatures. The granules at high $\mathrm{pH}$, on the other hand, show increasing growth rate parameters with increasing $\mathrm{pH}$ because the removal of lipids and proteins from the granules create surfaces where heterogeneous nucleation is more favorable. Referring to Figure 4, the heated granules were generally less than $2 \mu \mathrm{m}$ in diameter (Figure 4(a)), while the granules at high $\mathrm{pH}$ were generally less than $0.5 \mu \mathrm{m}$ in diameter (Figure 4(b)). The relative size of the PHB granules is dependent on the extent of particle aggregation, which occurs only at regions where PHB is amorphous and intermolecular entanglement can easily take place. With more surface area favorable for heterogeneous nucleation $\left(r_{n}>r_{c r}\right)$ than a sample of pure PHB, the frequency of nucleation in smaller PHB granules is much greater, resulting in larger values of the growth rate parameter.

\section{Conclusions}

The in situ crystallization of PHB in its native form is similar to that of pure PHB and can be modeled by Avrami's equation under isothermal conditions. The growth rate parameter $(k)$ is dependent on the crystallization environment (i.e., temperature and $\mathrm{pH}$ ) and describes the frequency of nucleation and subsequent spherulitic crystal growth. The Avrami exponent $(n)$ is dependent on the geometric nature of crystallization and is related to the number and size of nucleation points and spherulites.

In summary, the empirical values of $n$ and $k$ increase and decrease respectively, with the amount of available material (i.e., amount of PHB per crystal/granule) for nucleation and crystal growth. Pure PHB crystallized into large spherulitic structures from homogeneous nuclei $(n$ $=1.5010)$ at a low growth rate $(k=0.0118)$. PHB granules heated $\left(80-140^{\circ} \mathrm{C}\right)$ in acidic solution $(\mathrm{pH} 2)$ crystallized into partial spherulitic structures from heterogeneous nuclei $(n=0.2140)$ at moderate growth rates $(k=$ $0.0725-0.1215)$. PHB granules suspended in solutions of increasing $\mathrm{pH}(2-12)$ at room temperature crystallized instantaneously from heterogeneous nuclei at granule surfaces $(n=0)$ and high growth rates $(k=0.0428$ 0.7553).

\section{Acknowledgements}

This work is partially supported by the Consortium for Plant Biotechnology Research (CPBR) and the U.S. Department of Energy. 


\section{REFERENCES}

[1] W. D. Luzier, "Materials Derived from Biomass/BiodeGradable Materials," Proceedings of the National Academy of Sciences of the United States of America, Vol. 89, No. 3, 1992, pp. 839-842.

[2] A. J. Anderson and E. A. Dawes, "Occurrence, Metabolism, Metabolic Role, and Industrial Uses of Bacterial Polyhydroxyalkanoates," Microbiological Reviews, Vol. 54, No. 4, 1990, pp. 450-472.

[3] G. N. Barnard and J. K. M. Sanders, "The Poly- $\beta$-Hydroxybutyrate Granule in Vivo: A New Insight Based on Nmr Spectroscopy of Whole Cells," Journal of Biological Chemistry, Vol. 264, No. 6, 1989, pp. 3286-3291.

[4] S. Y. Lee, "Bacterial Polyhydroxyalkanoates," Biotechnology and Bioengineering, Vol. 49, No. 1, 1996, pp. 114.

[5] T. V. Ojumu, J. Yu and B. O. Solomon, "Production of Polyhydroxyalkanoates, a Bacterial Biodegradable Polymer," African Journal of Biotechnology, Vol. 3, No. 1, 2004, pp. 18-24.

[6] Y. Chen, J. Chen, C. Yu, G. Du and S. Lun, "Recovery of Poly-3-Hydroxybutyrate from Alcaligenes Eutrophus by Surfactant-Chelate Aqueous System," Process Biochemistry, Vol. 34, No. 2, 1999, pp. 153-157.

[7] J.-I. Choi and S. Y. Lee, "Efficient and Economical Recovery of Poly(3-Hydroxybutyrate) from Recombinant Escherichia Coli by Simple Digestion with Chemicals," Biotechnology and Bioengineering, Vol. 62, No. 5, 1999, pp. 546-553.

[8] J. S. Herron, J. D. King and D. C. White, "Recovery of Poly- $\beta$-Hydroxybutyrate from Estuarine Microflora," $A p$ plied and Environmental Microbiology, Vol. 35, No. 2, 1978, pp. 251-257.

[9] S. K. Hahn, Y. K. Chang and S. Y. Lee, "Recovery and Characterization of Poly(3-Hydroxybutyric Acid) Synthesized in Alcaligenes Eutrophus and Recombinant Escherichia coli," Applied and Environmental Microbiology, Vol. 61, No. 1, 1995, pp. 34-39.

[10] K. Sudesh, H. Abe and Y. Doi, "Synthesis, Structure And Properties of Polyhydroxyalkanoates: Biological Polyesters," Progress in Polymer Science, Vol. 25, No. 10, 2000, pp. 1503-1555.

[11] E. S. Stuart, A. Tehrani, H. E. Valentin, D. Dennis, R. W. Lenz and R. C. Fuller, "Protein Organization on the PHA Inclusion Cytoplasmic Boundary," Journal of Biotechnology, Vol. 64, No. 2-3, 1998, pp. 137-144.

[12] D. M. Horowitz and J. K. M. Sanders, "Amorphous, Biomimetic Granules of Polyhydroxybutyrate: Preparation, Characterization, and Biological Implications," Journal of the American Chemical Society, Vol. 116, No. 7, 1994, pp. 2695-2702.

[13] P. J. Barham, A. Keller, E. L. Otun and P. A. Holmes, "Crystallization and Morphology of a Bacterial Thermoplastic: Poly-3-Hydroxybutyrate," Journal of Materials Science, Vol. 19, No. 9, 1984, pp. 2781-2794.

[14] L. M. W. K. Gunaratne, R. A. Shanks and G. Amaras- inghe, "Thermal History Effects on Crystallisation and Melting of Poly(3-Hydroxybutyrate)," Thermochimica Acta, Vol. 423, No. 1-2, 2004, pp. 127-135.

[15] D. S. Conti, M. I. Yoshida, S. H. Pezzin and L. A. F. Coelho, "Miscibility and Crystallinity of Poly(3-Hy- Droxybtyrate)/Poly(3-Hydroxybutyrate-co-3-Hydroxyl-Valer ate) Blends," Thermochimica Acta, Vol. 450, 2006, pp. 61-66.

[16] J. Cornibert and R. H. Marchessault, "Conformational Isomorphism. A General 21 Helical Conformation for Poly(B-Alkanoates)," Macromolecules, Vol. 8, No. 3, 1975, pp. 296-305.

[17] A. Padermshoke, Y. Katsumoto, H. Sato, S. Ekgasit, I. Noda and Y. Ozaki, "Melting Behavior of Poly(3-Hydroxybutyrate) Investigated by Two-Dimensional Infrared Correlation Spectroscopy," Spectrochimica Acta Part A, Vol. 61, No. 4, 2005, pp. 541-550.

[18] H. Sato, R. Murakami, A. Padermshoke, F. Hirose, K. Senda, I. Noda and Y. Ozaki, "Infrared Spectroscopy Studies of $\mathrm{CH}^{\circ} \mathrm{O}$ Hydrogen Bondings and Thermal Behavior of Biodegradable Poly(Hydroxyalkanoate)," Macromolecules, Vol. 37, No. 19, 2004, pp. 7203-7213.

[19] W. D. J. Callister, "Materials Science and Engineering: An Introduction," John Wiley \& Sons, Inc., Hoboken, 2003.

[20] P. J. Barham, "Nucleation Behaviour of Poly-3-HydroxyButyrate," Journal of Materials Science, Vol. 19, No. 12, 1984, pp. 3826-3834.

[21] J. Zhang, H. Sato, I. Noda and Y. Ozaki, "Conformation Rearrangement and Molecular Dynamics of Poly(3-Hydroxybutyrate) During the Melt-Crystallization Process Investigated by Infrared and Two-Dimensional Infrared Correlation Spectroscopy," Macromolecules, Vol. 38, No. 10, 2005, pp. 4274-4281.

[22] T. Furukawa, H. Sato, R. Murakami, J. Zhang, Y.-X. Duan, I. Noda, S. Ochiai and Y. Ozaki, "Structure, Dispersibility, and Crystallinity of Poly(Hydroxybutyrate)/ Poly(L-Lactic Acid) Blends Studied by FT-IR Microspectroscopy and Differential Scanning Calorimetry," in Macromolecules, Vol. 38, No. 15, 2005, pp. 6445- 6454.

[23] A. S. Myerson, "Molecular Modeling Applications In Crystallization," Cambridge University Press, Cambridge, 1999. doi:10.1017/CBO9780511529610

[24] M. D. Ward, "Bulk Crystals To Surfaces: Combining XRay Diffraction and Atomic Force Microscopy to Probe the Structure and Formation of Crystal Interfaces,' Chemical Reviews, Vol. 101, No. 6, 2001, pp. 1697-1726.

[25] K. Grage, A. C. Jahns, N. Parlane, R. Palanisamy, I. A. Rasiah, J. A. Atwood and B. H. A. Rehm, "Bacterial Polyhydroxyalkanoate Granules: Biogenesis, Structure, and Potential Use as Nano-/Micro-Beads in Biotechnological and Biomedical Applications," Biomacromolecules, Vol. 10, No. 4, 2009, pp. 660-669.

[26] S. Bloembergen, D. A. Holden, G. K. Hamer, T. L. Bluhm and R. H. Marchessault, "Studies of Composition and Crystallinity of Bacterial $\operatorname{Poly}(\beta$-Hydroxybutyrateco- $\beta$-Hydroxyvalerate)," Macromolecules, Vol. 19, No. 
11, 1986, pp. 2865-2871.

[27] A. Paermshoke, Y. Katsumoto, H. Sato, S. Ekgasit, I. Noda and Y. Ozaki, "Melting Behavior of Poly(3-Hydroxybutyrate) Investigated by Two-Dimensional Infrared Correlation Spectroscopy," Spectrochimica Acta Part A, Vol. 61, No. 4, 2005, pp. 541-550.

[28] J. Xu, B.-H. Guo, R. Yang, Q. Wu, G.-Q. Chen and Z.-M. Zhang, "In situ FTIR Study on Melting and Crystallization of Polyhydroxyalkanoates," Polymer, Vol. 43, No. 25, 2002, pp. 6893-6899.

[29] J. Li, C. Zhou, G. Wang, Y. Tao, Q. Liu and Y. Li, "Isothermal and Nonisothermal Crystallization Kinetics of
Elastomeric Polypropylene," Polymer Testing, Vol. 21, No. 5, 2002, pp. 583-589.

[30] M. Liu, Q. Zhao, Y. Wang, C. Zhang, Z. Mo and S. Cao, "Melting Behaviors, Isothermal and Non-Isothermal Crystallization Kinetics of Nylon 1212," Polymer, Vol. 44, No. 8, 2003, pp. 2537-2545.

[31] Y. An, L. Dong, L. Li, Z. Mo and Z. Feng, "Isothermal Crystallization Kinetics and Melting Behavior of Poly(BHydroxybutyrate)/Poly(Vinyl Acetate) Blends," European Polymer Journal, Vol. 35, No. 3, 1999, pp. 365-369. 